\title{
DIFICULTADES PARA LA FORMULACIÓN DE UNA TEORÍA EN TORNO AL ESTADO DE LAS AUTONOMÍAS
}

MANUEL FONDEVILA MARÓN 
SUMARIO

I. INTRODUCCIÓN: CONTEXTO SOCIO-POLÍTICO EN EL QUE SE ENMARCA LA OBRA. II. EL ESTADO DE LAS AUTONOMÍAS Y LA INDETERMINACIÓN DE LA FORMA DE ESTADO EN LA CONSTITUCIÓN DE 1978. III. SOBRE LA CARACTERIZACIÓN DE LOS ELEMENTOS QUE CONFORMAN EL TIPO «ESTADO FEDERAL». IV. SOBERANÍA Y AUTONOMÍA, O SOBRE LA DISTRIBUCIÓN TERRITORIAL Y FUNCIONAL DEL PODER PÚBLICO COMO CARACTERÍSTICA BÁSICA DEL TIPO ESTADO FEDERAL. V. DE LA KOMPETENZ-KOMPETENZ Y SUS LÍMITES. VI. CONCLUSIÓN. 


\title{
DIFICULTADES PARA LA FORMULACIÓN DE UNA TEORÍA EN TORNO AL ESTADO DE LAS AUTONOMÍAS
}

\section{(A PROPÓSITO DE «DIVISIÓN DE COMPETENCIAS Y FORMA TERRITORIAL DEL ESTADO» DE JAVIER RUIPÉREZ ALAMILLO)}

\author{
MANUEL FONDEVILA MARÓN \\ Investigador \\ Universidade da Coruña
}

I. INTRODUCCIÓN: CONTEXTO SOCIO-POLÍTICO EN EL QUE SE ENMARCA LA OBRA

Unas palabras preliminares acerca de la obra que en este trabajo presentamos y analizamos resultan, a nuestro juicio, necesarias. Ello porque en España se ha planteado un problema de índole político y jurídico de enorme trascendencia constitucional. Nos referimos evidentemente al desafío que el Presidente de CIU y de la Generalitat de Catalunya, Sr. Artur Mas, plantea al conjunto del Estado cuando afirma que convocará, en la legislatura que comenzó tras las elecciones del 25 de Noviembre de 2012, una consulta popular en la que preguntará al electorado si desea que Cataluña se establezca como un Estado independiente dentro de la Unión Europea y, caso de ser la respuesta afirmativa, que llevará a 
cabo el proceso, sin que ningún Tribunal o Constitución puedan oponerse ${ }^{1}$. Lo hace, a pesar de que sabe, o debe saber, que el artículo 149.1.32 de la Constitución española establece la competencia exclusiva del Estado para autorizar consultas populares, y que el Tribunal Constitucional, intérprete supremo de la Constitución, ya anuló una pretensión similar del gobierno vasco en fechas relativamente recientes ${ }^{2}$. Lo hace, también, a pesar de que sabe, o debe saber, que su propuesta de un Estado independiente en la Unión Europea es imposible jurídicamente hablando, al menos por dos razones: En primer lugar, porque la Unión Europea, tal y como indican los Tratados Constitutivos, respeta las funciones esenciales de los Estados, y entre ellas, la integridad territorial de los mismos $^{3}$ y, en segundo lugar, porque dado que el único sujeto, actualmente, de Derecho Comunitario europeo es el Reino de España ${ }^{4}$, la adhesión de un nuevo Estado tendría que contar con la unanimidad de todos los Estados miembros del Consejo de la Unión Europea, incluida España. Además, y por cuanto a la secesión unilateral de una Comunidad Autónoma, o de cualquier ente territorial se refiere, ocioso debiera resultar indicar que es, obviamente, una posibilidad tajantemente prohibida por nuestra Carta Fundamental de 1978. Por lo tanto, y en conclusión, nos encontramos en una situación en la que se plantea, por parte del Presidente de una Comunidad Autónoma, de forma ilegal, una pregunta cuya respuesta sobrepasa el halo de competencias del gobierno que preside.

Más importancia, sin embargo, que este problema, coyuntural en el tiempo, y que por lo que sintéticamente acabamos de exponer está destinado al fracaso sin paliativos, es indicar que, al menos desde el comienzo del siglo XXI, tanto a nivel comunitario como a nivel infraestatal se están produciendo acontecimientos que exigen: en primer lugar, un conocimiento riguroso sobre las posibilidades que cada tipo de forma política (Confederación, Estado Federal, Estado Unitario) permite en cuanto a distribución de competencias; en segundo lugar, en quién reside, en última instancia, la soberanía en cada uno de estas tres tipologías estatales; y, por último, en que se diferencia este concepto de soberanía de otros como es el de la «competencia sobre la competencia». Sin ánimo de exhaustividad, los hechos a los que nos referimos son los siguientes: a) Entre los años 2003 y 2004 se debatió y propuso la aprobación de un Tratado por el que se establecía una «Constitución para Europa. b) Habiendo sido abandonado el

${ }^{1}$ Sobre este tema RUIPÉREZ ALAMILLO, J. «La nueva reivindicación de la Secesión de Cataluña en el contexto normativo de la Constitución española de 1978 y el Tratado de Lisboa». En Teoría y Realidad Constitucional. n. ${ }^{\circ} 31$ (2013).

2 Vid. STC 103/2008.

3 Vid. art. 4 TUE.

${ }^{4}$ Vid. Preámbulo del TUE. 
Proyecto de Constitución, debido al rechazo en referéndum por parte del pueblo francés y holandés, a partir de 2007 las instituciones europeas trabajaron en un nuevo Tratado, el Tratado de Lisboa, que entró en vigor en 2009, que respeta, en esencia, el espíritu de aquella Constitución ${ }^{5}$, ampliando sustancialmente tanto las materias competencias de la Unión como aquellas materias en las que la decisión comunitaria se puede adoptar por mayoría en lugar de unanimidad. c) En Italia, una serie de reformas constitucionales llevadas a cabo en 1999 y $2001^{6}$ permitió a las Regiones, sobre todo las de Estatuto ordinario, ampliar, por vía de la reforma de estos Estatutos, enormemente sus competencias. d) Lo mismo ha ocurrido en España con la aprobación de los que podríamos calificar «Estatutos de segunda generación» aprobados en algunas Comunidades Autónomas (Comunidad Valenciana, Cataluña, Islas Baleares, Andalucía, Aragón y Castilla y León) ${ }^{7}$ e) Lo mismo ha ocurrido desde $1998^{8}$ en Gran Bretaña, iniciándose un proceso conocido como «devolution» que aún hoy en día es difícil saber como terminará, teniendo en cuenta que está previsto un referéndum independentista en Escocia para 2014.

La virtualidad, por lo tanto, de la obra del Profesor J. Ruipérez es precisamente dar respuesta a los interrogantes a los que nos acabamos de referir en un contexto socio-político como el actual. No es la primera vez que el autor se plantea estas cuestiones. De hecho, el problema del procedimiento a seguir para llevar a cabo la secesión de un territorio se lo planteaba ya, al menos de un modo hipotético en su trabajo titulado Constitución y Autodeterminación (1995), en la Protección Constitucional de la Autonomía (1994) ya sostuvo que este elemento era precisamente el que caracterizaba a un Estado federal o políticamente descentralizado y, finalmente, en la recopilación de trabajos bajo el título Proceso Constituyente, Soberanía y Autodeterminación (2003) se trata el tema de la soberanía en el Estado de las Autonomías. En el trabajo que ahora comentamos aborda estos problemas pero de una manera diferente: se trata de una obra no sólo más breve sino mucho más concreta, en la que tal y como iremos comentando en las pági-

${ }^{5}$ Vid., por todos, BAR CENDÓN, A. «La nueva Constitución de la Unión Europea: El Tratado de Lisboa y la Reforma de los Tratados Constitutivos». En Teoría y Realidad Constitucional. N. 25 (2010), y MARTÍN Y PÉREZ DE NANCLARES, J. «Hacia un nuevo Tratado europeo: de cómo recuperar la esencia del Tratado Constitucional sin que realmente lo parezca» (2007). Análisis para el Real Instituto Elcano, consultable a través de Internet.

${ }^{6}$ Vid. L. Cost. 1/1999, 2/2001 y 3/2001.

7 Para mayor profundización se puede consultar: FONDEVILA MARÓN, M. «Los Nuevos Estatutos de Autonomía en el Marco del Derecho Constitucional Comparado» en Boletín Mexicano de Derecho Comparado. N. ${ }^{\circ} 133$ (2012). pp. 13-54.

8 Vid. Scotland Act (1998, modificada en 2012), Northern Ireland Act (1998-2009) y Goverment of Wales Act (2006). 
nas que siguen, critica nuestro autor, con extraordinario rigor científico, las clasificaciones al uso de los elementos que caracterizan un Estado federal o políticamente descentralizado, indicando a quién corresponde, en este tipo de Estado, la competencia sobre la competencia, y los límites materiales inherentes a la misma.

Vamos a comentar, a continuación, cada uno de los aspectos mencionados. Sin embargo, con carácter previo queremos nosotros dedicar unas palabras a exponer lo que, a nuestro juicio, es la problemática fundamental del Estado de las Autonomías: la dificultad que un, sin duda, desafortunado Título VIII de la Constitución, ha supuesto, para académicos y prácticos de la Política y el Derecho, a la hora de definir y caracterizar la forma del Estado español.

\section{EL ESTADO DE LAS AUTONOMÍAS Y LA INDETERMINACIÓN DE LA FORMA DE ESTADO EN LA CONSTITUCIÓN DE 1978}

Escribía el Profesor P. de Vega, en el prólogo de otra obra con la que el Profesor J. Ruipérez iniciaba el estudio de esta problemática, todavía no resuelta, en torno a la forma territorial del Estado de las Autonomías, que: «Las vacilaciones y condicionamientos que en momento constituyente condujeron a una inacabada construcción del Estado de las Autonomías, y que se plasmaron en el infeliz y problemático Título VIII de la Constitución, forzosamente tenían que repercutir en la forja de la dogmática jurídica del Estado Autonómico, que, indefinido e inconcluso en sus planteamientos y presupuestos político-constitucionales, necesariamente tenía que resultar dubitativo y proteico en muchas de sus formulaciones dogmáticas» ${ }^{9}$. Al menos, es evidente que no podrá sostenerse la dogmática a la que el Profesor Emérito de la Universidad Complutense hacía referencia desde planteamientos positivistas y formalistas — tal y como con gran brillantez e ironía puso de manifiesto, en los albores del Estado Autonómico, el Profesor P. Cruz ${ }^{10}$ — pues de lo que 35 años después de haberse aprobado la Constitución de 1978 no puede caber duda es que la distribución de competencias dista mucho de tener una regulación satisfactoria. No creemos — tal y como ha insinuado nuestro Tribunal Constitucional (volveremos sobre ello más

9 DE VEGA, P. «prólogo» en RUIPÉREZ ALAMILLO, J. La Reforma del Estatuto de Autonomía para Galicia. Universidade da Coruña. 1995. p. 10.

${ }^{10}$ Cfr., Cruz Villalón, $\mathrm{P}$ «La estructura del Estado o la curiosidad del jurista persa», en La Curiosidad del Jurista Persa, y Otros Escritos sobre la Constitución, Centro de Estudios Políticos y Constitucionales, Madrid, 1999 —originariamente, 1982_, pp. 381 a 394. 
adelante)— que sea una materia «desconstitucionalizada», pero sí que requiere mucha más concreción y delimitación.

Una prueba, a nuestro juicio tan contundente como actual, de la confusión que existe en la doctrina como en la práctica política en torno a la forma territorial del Estado español es la respuesta por parte del Partido Socialista Obrero Español (asesorado por juristas de reconocida competencia en Derecho Público), ante los planteamientos soberanistas del partido nacionalista de índole regional que gobierna en Cataluña, de reformar la Constitución para hacer de España «Un Estado Federal». En un documento que se titula «Por una Reforma Federal del Estado Autonómico», a pesar de que se reconoce que el modelo autonómico se incardina en la matriz de los Estados federales se afirma, sin embargo, que no es un Estado puramente federal por no existir voluntad constituyente expresa y faltar, en consecuencia, plenitud y legitimidad. A nuestro juicio de esta manera se confunden las cosas. Cualquiera puede ver, simplificando, que lo que proponen es una reforma del Estado Federal actualmente existente. Una reforma con la que, en sus aspectos concretos, se puede estar más o menos de acuerdo, pero que en sí no modificaría para nada la naturaleza descentralizada de la España que configuró la Constitución de 1978.

En los primeros años de andadura del Estado Autonómico, y dado que el Título VIII de la Constitución de 1978 así como algunas disposiciones transitorias (que, por cierto, tienen poco sentido en el momento actual) regulaban el acceso a la autonomía de los entes territoriales formados por provincias tenía aún sentido hablar del «Estado Autonómico» (formula, además, tan ausente en el articulado del texto constitucional como la de Estado federal) como asimilable al «Estado Regional» italiano en el que parecía inspirarse, al menos por lo que respecta a los dos tipos de entidades territoriales, unos con más autonomía que otros. Hoy en día, sin embargo, y después de que en 1992 se alcanzara un techo competencial idéntico para todas las Comunidades Autónomas, y en 1999 y 2001 (tal y como hemos mencionado), tampoco pueda decirse ya que existan diferencias institucionales entre las Regiones de Estatuto Ordinario y Especial, podemos sentar la tesis de que estos dos Estados, de los que acaso podría haber, en su origen, duda ${ }^{11}$ acerca de su naturaleza federal — debido a la quiebra del principio de igualdad federal-, han evolucionado formando parte ahora, de modo evidente, del tipo Estado federal.

${ }^{11}$ El Profesor J. Ruipérez, sin embargo, defendía ya entonces como ahora que no cabía defender que el Estado Regional o el Estado Autonómico fueran una categoría distinta y equidistante entre el Estado Unitario y Estado Federal (Véase el Estudio Preliminar del Código de Derecho Constitucional Autonómico, en Biblioteca Nueva). 
En España se ha dado, de hecho, una situación paradójica: se ha pasado de entender que el Estado de las Autonomías español, aun cuando estuviese inspirado, como hemos dicho, en el Estado Regional italiano, no llegaba tan lejos como éste, siendo una suerte de Estado Unitario administrativamente descentralizado excepto para las Comunidades Autónomas que accediesen a la autonomía por la vía del art. 151, que podrían disponer de un poder legislativo propio, ostentando por ello una legitimidad democrática también propia, a considerar que la Constitución de 1978 permite adoptar cualquier tipo de reparto territorial de poder entre Estado y Comunidades Autónomas, incluso uno más propio de una organización de Derecho Internacional, como es una Confederación de Estados, que de un verdadero y auténtico Estado Federal ${ }^{12}$.

Sin embargo, lo más grave no es que existan dudas y vacilaciones entre la doctrina a la hora de definir la forma territorial del Estado. La doctrina no es fuente del Derecho ${ }^{13} \mathrm{y}$, siendo además numerosos los estudiosos de las Ciencias Constitucionales que se han dedicado a esta cuestión, es evidente que hay —en nuestra humilde opinión - algunos que lo han hecho con mayor precisión que otros. Por eso, simplemente nos interesa destacar que existen quienes, como el Profesor J. Pérez Royo de quien, desde el mayor de los respetos, discrepamos, afirman que es a través del Estatuto de Autonomía, de cada uno de ellos en particular y de todos en su conjunto, con el que se da forma al Estado Autonómico (razón por la cual, en su opinión, no puede haber más guardián de su constitucionalidad que las Cortes Generales, en el trámite de su aprobación como Ley Orgánica $)^{14}$, conducen lógicamente a la conclusión de que siempre que exista acuerdo entre las Cortes y la Asamblea Legislativa de la Comunidad Autónoma los Estatutos podrán contener cualesquiera disposiciones, que muy difícilmente podrán considerarse inconstitucionales, dado que son ellos mismos los que determinan la naturaleza de la forma de Estado. No se trata de un problema nuevo, ni de un estado de opinión actual: en 1995, en el prólogo citado, afirmaba también el Profesor P. de Vega que no sabría pronunciarse «sobre si la conversión del momento estatuyente en momento constituyente, o la utilización de categorías como el "bloque de constitucionalidad" son geniales creaciones de la ingeniería jurídica constitucional, o simples adefesios de juristas al servicio del

${ }^{12}$ Cfr., RUIPÉREZ ALAMILLO, J. Entre el Federalismo y el Confederantismo. Dificultades y problemas para la formulación de una teoría constitucional del Estado de las Autonomías. Biblioteca Nueva. Madrid 2010. pp. 85 a 189.

13 Vid., art. 1 CC.

${ }^{14}$ Cfr., PEREZ ROYO, J. «Por qué el Tribunal Constitucional no puede ser el guardián de la constitucionalidad de los Estatutos de Autonomía». En Teoría de la Constitución. Estudios jurídicos en homenaje al Dr. Carpizo en Madrid. Porrua. México. 2010. p. 592. 
pragmatismo político más grosero y vulgar» ${ }^{15}$. A fin de cuentas lo que se hace con construcciones doctrinales de este estilo no es más que conceder a las Comunidades Autónomas la co-titularidad del ejercicio de la kompetenz-kompetenz, que tal y como veremos en el epígrafe $\mathrm{V}$ de este trabajo, aun en los Estados federales, corresponde únicamente al poder central.

Lo más grave es, por el contrario, que tesis por el estilo se deduzcan de la jurisprudencia del máximo intérprete constitucional. No es sólo, como vamos a sostener también en el epígrafe $\mathrm{V}$, al que nos remitimos, que la jurisprudencia reciente del Tribunal Constitucional nos parezca criticable. En un orden de consideraciones anterior y general habría que denunciar, también con el Profesor P. De Vega, en este caso en el prólogo con ocasión de uno de los libros de quien igualmente ha sido Profesor de quien escribe estas líneas en la Universidad de La Coruña, que «la conversión subrepticia del Tribunal Constitucional en una especie de Poder Constituyente permanente, sitúa la problemática jurídica sobre el mismo en un ámbito en el que los planteamientos jurídicos no pueden realizarse al margen de los efectos indiscutiblemente políticos de sus sentencias» ${ }^{16}$. Por eso no cabe extrañarse de que haya habido quien, tal y como acabamos de ver, haya defendido que el Tribunal Constitucional carecía de competencia para enjuiciar la constitucionalidad de los Estatutos de Autonomía. En todo caso, hoy es evidente que la carga de haber tenido que enjuiciar la constitucionalidad del Estatuto de Autonomía de Cataluña ha resultado excesivamente pesada para el Tribunal.

En la era de la postmodernidad, donde resulta verdaderamente difícil la construcción de teorías generales, tampoco es de extrañar, y con una breve referencia a ello terminamos este epígrafe, que dudas y vacilaciones, en la doctrina y en la jurisprudencia acerca de la forma política, se reproduzcan en el ámbito de una Unión Europea actualmente a caballo entre la Confederación de Estados, tal y como la denominó en su día A. La Pérgola ${ }^{17}$ y un verdadero Estado Federal. Sin ánimo de exhaustividad podemos afirmar que estas dudas y vacilaciones repercuten en, al menos, dos aspectos suficientemente relevantes: En primer lugar, sobre los Derechos Fundamentales. La Carta de Niza, desde la entrada en vigor del Tratado de Lisboa con valor jurídico vinculante, presenta problemas interpretativos en aquellas áreas, cada vez más numerosas, en las que la delimi-

15 DE VEGA, p. óp. cit. p.12.

16 DE VEGA, P. «Prólogo» EN ROURA GOMEZ, S. Federalismo y Justicia Constitucional en la Constitución Española de 1978. Biblioteca Nueva. Madrid 2003. p. 17.

17 Cfr., LA PERGOLA, A. «Sguardo sul federalismo e i suoi dintorni (una célebre dicotomía: «stato federale-confederazione», La Confederazione di tipo antico e moderno. L'idea europeistica di comunità). En Diritto e Societá. 1992 (3). pp. 500 y ss. 
tación competencial entre los Estados y la Unión resulta algo difusa, sobre todo en atención a lo dispuesto en el art. 51.2 de la misma ${ }^{18}$. En segundo lugar, los principios de primacía y de subsidiariedad, interpretados por el Tribunal de Justicia de un modo tan expansivo que el significado del primero de ellos se aleja del que habitualmente tiene en el Derecho Internacional y se asemeja más al de, por ejemplo, el art. 31 de la Ley Fundamental de Bonn, mientras que el segundo de ellos, interpretado de manera laxa de modo que permite a la Unión justificar su acción sobre una materia con una mera declaración formal de que no conviene hacerlo en un nivel inferior es, como agudamente ha sostenido la Profesora M. Rodríguez Izquierdo, una manifestación más de primacía comprendida con una óptica federal ${ }^{19}$.

Así las cosas, creemos que se comprende con facilidad la pertinencia y necesidad de la obra del Profesor J. Ruipérez a propósito de la cual escribimos este ensayo, ampliándolo a partir de ahora al hilo de las principales aportaciones de la misma.

\section{SOBRE LA CARACTERIZACIÓN DE LOS ELEMENTOS QUE CONFORMAN EL TIPO «ESTADO FEDERAL»}

La labor del iuscomparatista se basa en buena medida en establecer clasificaciones o conceptos en base a unas características objetivas ${ }^{20}$. El problema de esta técnica puede ser que cada autor comparatista elabore sus propias familias jurídicas, conforme a los elementos definidores que él considera más relevantes, lo cuál no tiene necesariamente que ser compartido por otros académicos. Precisamente esto es lo que, en concreto, denuncia que ocurre A. La Pérgola respecto de los elementos normalmente considerados definidores de la forma Estado Federal. En su idea, así como en las tesis de Ch. Durand se basa nuestro autor para hacer una crítica a la clasificación, de K. Wheare ${ }^{21}$. Clasificación, por lo demás, muy generalizada, en el Derecho Constitucional Comparado. Entre otros

18 Para esto, por comodidad y brevedad me remito a FONDEVILA MARÓN, M. «El Modelo Confederal e Imperfecto de Protección de Derechos Fundamentales en la UE». En Revista de Derecho de la UNED. N. ${ }^{\circ} 11$ (2012).

${ }^{19}$ Cfr., RODRÍGUEZ-IZQUIERDO SERRANO, M. Primacía y Subsidiariedad en la Unión Europea. CEPC. Madrid, 2011.

${ }^{20}$ Cfr., PEGORARO, L. y RINELlA, A. Diritto Pubblico Comparato. Profili Metodologici. CEDAM. Padova. 2007. p. 45.

${ }^{21}$ Cfr., WHEARE, K. Govern Federal. Institut d’Estudis Autonomics. Traducció Dolors Udina. 2008. pp. 167-196. 
muchos, podemos citar los conocidos trabajos de R. Watts ${ }^{22}$ y D. Elazar ${ }^{23}$ así como otros trabajos más recientes de autores como H-P Schneider ${ }^{24}$. Los elementos que contienen la clasificación de estos autores son los siguientes:

a) Dos niveles de gobierno, actuando cada uno de ellos directamente sobre los ciudadanos.

b) Una división constitucional formal de poderes legislativo, ejecutivo y judicial, y una asignación de ingresos y recursos entre los dos niveles de gobierno, garantizando algunas áreas de genuina autonomía para cada nivel de gobierno.

c) Disposiciones para integrar la representación de los distintos entes regionales en la elaboración de políticas de las instituciones federales, normalmente prevista a través de una forma particular de segunda cámara federal.

d) Una suprema constitución escrita no reformable unilateralmente, y que requiere para su reforma el consentimiento de la mayoría de las unidades constituidas o de sus representantes en la segunda cámara.

e) Un árbitro (en la forma de corte de justicia o previsión de referéndum) para mediar en las disputas entre los gobiernos.

f) Procedimientos e Instituciones para facilitar la colaboración intergubernamental en los ámbitos donde comparten responsabilidad gubernamental o donde las responsabilidades inevitablemente se solapan.

Ahora bien, otros reputados autores manejan clasificaciones mucho más simplificadas y, a nuestro juicio, acertadas. Por ejemplo, el Profesor A. Reposo ${ }^{25}$ nos ofrece una clasificación aún más simplificada de lo que él llama «Estado Autonómico» (que incluye tanto lo que habitualmente se considera en la doctrina Estado Federal como Estado Regional) que se compondría de, simplemente:

a) La existencia de distintos niveles de gobierno.

b) Una división de competencias en tres órdenes.

c) Una constitución escrita y rígida.

${ }^{22}$ Cfr., WATTS, R. L. Sistemas Federales Comparados. Traducción de Esther Seijas. Marcial Pons. Madrid-Barcelona 2006. p. 106.

${ }^{23}$ Cfr., ELAZAR, DJ. Exploración del Federalismo. Traducción de Josep M. ${ }^{a}$ Sole Alseda. Hacer. Barcelona 1990. p. 201.

${ }^{24}$ Cfr. SCHNEIDER, H-P. «Unitary and Federal States: Historical and Political Perspectives». En Ferrer McGregor y Zaldivar Lelo de Lárrea, A, La Ciencia del Derecho Procesal Constitucional. Estudios en Homenaje a Fix-Zamudio en sus cincuenta años como investigador del Derecho Tomo XI. Marcial Pons. México 2008. pp. 229-245.

25 REPOSO, A. Profili dello Stato Autonómico. Federalismo e regionalismo. Giappichelli. Torino. 2005. p. 91. 
Tanto nosotros como el autor cuya obra estamos comentando compartimos esta clasificación de elementos caracterizadores de un Estado Federal. Ahora bien, sobre el tercero de los elementos es menester una breve aclaración pues el Profesor J. Ruipérez, como la mayoría de la doctrina, comparte esta concepción según la cuál la existencia de una Constitución formal, escrita y rígida es indispensable para poder hablar de Estado federal. De hecho, afirma literalmente: «Debe darse, entonces, la razón a Friedrich cuando señala que el Estado Políticamente Descentralizado sólo funcionará de un modo cabal y ponderado cuando la técnica de reparto territorial y funcional del poder político se encuentre incorporado al Derecho. De una manera más concreta, cuando la distribución competencial que tan sólo puede ser decretada por el Pouvoir Constituant, venga establecida en el texto de una Constitución escrita, formal, solemne de carácter rígido. Lo que, ni que decir tiene, excluye la actuación en este campo de los poderes constituidos federales y regionales ${ }^{26}$. Pero para interpretar correctamente sus palabras debe tenerse en cuenta $-\mathrm{y}$ la pista nos la da el que se esté remitiendo a Friedrichque por constitución escrita y rígida no se está refiriendo a lo que por ello entendían, por ejemplo Preuss o el propio K. Wheare ${ }^{27}$, esto es, un documento formal que contiene un procedimiento de reforma mucho agravado que el previsto para las leyes ordinarias, sino lo que, efectivamente, atendiendo a la realidad política y social de un Estado concreto, pueda considerarse una Constitución difícilmente reformable. De este modo es claro para nosotros que un Estado como es la Gran Bretaña - a cuya situación política nos hemos referido ya en la Introducción- debería incluirse dentro de los Estados Federales o Políticamente descentralizados, pues —en contra de lo que algunos autores, como el Profesor J. J. Solozábal, para quien el Parlamento de Westminster puede mañana, por ejemplo, legislar sobre las materias que no se ha reservado en la Scotland Act o, como ha sucedido en varias ocasiones en el caso de Irlanda, incluso decidir suprimir la autonomía de cualquiera de las tres regiones (Escocia, Gales, e Irlanda del Norte) a las que ha traspasado competencias de gobierno ${ }^{28}$ — se nos antoja tan difícil que la devolution tenga marcha atrás como que lo tenga el Estado federal alemán, el Estado regional italiano, o el Estado autonómico español.

De esto no se deduce, sin embargo, que necesariamente deban los entes federados de un Estado Políticamente descentralizado participar o autorizar una posible reforma de la Constitución. La razón de porqué nos detenemos en esta

${ }^{26}$ RUIPÉREZ ALAMILLO, J. División de Competencias y Forma Territorial del Estado. Reus. Madrid, 2012. p. 49.

${ }^{27}$ Cfr., WHEARE, K. óp. cit. pp. 433 y ss.

${ }^{28}$ Cfr. SOLOZABAL ECHABARRÍA, J. J. «Sobre la Devolution en el Reino Unido (Letter from London)» en Revista General de Derecho Constitucional. n. ${ }^{\circ} 1$ (2006) p. 5. 
cuestión es que, incluso un académico como el Profesor L. Pegoraro, muy cercano a nuestra tesis acerca de la naturaleza del Estado Federal cuando afirma que «[en la actualidad] entra en crisis, la definición misma de "Estado Federal", con la cual no puede definirse más un "Estado de Estados" provisto de soberanía interna y externa, sino un ordenamiento ampliamente descentralizado» ${ }^{29}$ afirma luego, en la misma obra, sin embargo, que quizás la única diferencia cualitativa entre Estado Federal y Regional sea, quizás, la participación de los entes federados en el proceso de revisión constitucional ${ }^{30}$. Que la responsabilidad de la reforma constitucional en el sentido de poder alterar, dentro del juego habitual de fuerzas centrífugas y centrípetas que tiene lugar en cualquier Estado federal corresponde a los órganos de la federación es algo sobre lo que vamos a tratar ampliamente en el penúltimo epígrafe de este trabajo, aun cuando en este momento, queremos introducir dos consideraciones críticas en refutación de la tesis contraria. En primer lugar, creemos que la afirmación, ausente de mayores matices, ignora una distinción fundamental entre revisión y destrucción de la Constitución ${ }^{31}$. Lo importante es tener en cuenta que, si la razón por la que se sostiene esto, es que no parece lógico considerar Estado Federal a un Estado donde desde los órganos centrales se pueda hacer desaparecer la autonomía política de los entes federados, en primer lugar, no se trataría de una simple revisión constitucional (pues es fácil comprender que una España o Italia unitarias no serían las mismas que vieron la luz tras las Constituciones de 1978 y 1947 respectivamente). En segundo lugar, y cuando se atiende a la realidad social de los Estados Políticamente descentralizados, creemos que sólo el entrañable, positivista, y esperpéntico jurista persa con el que ironizaba el Profesor P. Cruz puede creer, en atención a la realidad jurídico-política de estos Estados, que desde Madrid y Roma se podría orquestar una modificación de la Constitución (por

${ }^{29}$ Cfr. PEgOrARO, L. La Costituzione brasiliana di 1998. CCSDD. Bologna. 2008. p. 30. Incluso, podría ser mejor afirmar como hace R. Blanco Valdes, que «el federalismo no existe» y que lo que queda, por tanto, es reconocer distintos tipos de federalismo allí donde encontremos una división del poder político sobre el territorio (Cfr. BLANCO VALDES, R. «La Spagna, el federalismo e Godot» en Politica del diritto. 2006 (2) pp. 321-336.

30 Ibídem. p. 33.

31 Por razones de espacio y sistematicidad, no podemos ahora detenernos en esta cuestión demasiado, y nos remitimos, con carácter general, a los siguientes trabajos: DE VEGA, P. La Reforma Constitucional y la Problemática del Poder Constituyente. Tecnos. Madrid 1991. RUIPÉREZ ALAMILLO, J. «Estática y dinámica constitucionales en la España de 1978. Especial referencia a la problemática de los límites a los cambios constitucionales». En ROURA, S y TAJADURA, J. (dirs). La Reforma Constitucional. Biblioteca Nueva. Madrid 2004. NOGUEIRA ALCALÁ, H. «Consideraciones sobre el poder constituyente y reforma de la Constitución en la teoría y práctica constitucional». En Revista Ius et Praxis. Año 15 (2008). N. ${ }^{\circ}$ 1. pp. 229-263. 
más que la Constitución española de 1978 prevea, en su artículo168 la «reforma total» y la Constitución Italiana sólo haga recaer la cláusula de intangibilidad sobre la forma republicana de Estado, en su artículo 139), que implique la vuelta a un Estado unitario ${ }^{32}$.

Aclarado este extremo comentamos los otros elementos que aparecen en las clasificaciones basadas en las de $\mathrm{K}$. Wheare.

Sobre la cámara de representación territorial, en concreto, sostiene el Profesor J. Ruipérez, de la mano, como hemos dicho, de Ch. Durand y A. La Pérgola que porque el Estado Federal no consiste, a diferencia de lo que ocurre en la Confederación de Estados, en aunar las voluntades de distintos entes soberanos (puesto que existe un único soberano, que es el Pueblo federal), evidente resulta que «estas Cámaras de representación territorial constituyen unos magníficos e innegables ejemplos de lo que [A. La Pérgola] considera «residuos contractualísticos» en el Estado Constituant federal, quien, no obstante su naturaleza de poder absoluto, soberano e ilimitado en el contenido de su voluntad se encuentra condicionado por la situación existente en la anterior etapa confederal» ${ }^{33}$. No le falta razón, efectivamente, a nuestro autor cuando indica que la argumentación al uso para defender el Senado como Cámara de Representación Territorial como la forma en que los entes federados recuperan un poco del poder público que habían perdido al ceder su soberanía a la federación puede servir, efectivamente, para explicar una federación que surge por la Unión de Estados antes soberanos pero que, en cambio, difícilmente puede explicar el surgimiento de federaciones consecuencia de un proceso de descentralización de Estados otrora unitarios. No le falta, de hecho, razón al Profesor J. Ruipérez cuando se pregunta cómo puede «recuperarse» aquello que nunca se ha tenido. Sin embargo, el doble tipo de formación histórica de Estados federales está también generalizada por la doctrina, que hoy rara vez sostiene que la Unión de Estados sea la única forma posible de surgimiento de un Estado federal y entendiendo el federalismo como un proceso (Fiedrich) distinguen, a nuestro modo de ver acertadamente, entre federalismo agregativo y federalismo disgregativo ${ }^{34}$. En este segundo tipo de Estados federales podríamos encontrar Estados como por ejemplo

32 Si de lo que se trata es, como afirmó ya en 1939 G. Lucatello (Cfr. LUCATELLO, G. Lo Stato Federale. CEDAM. Padova. 1967. P. 188) de que los entes federados participen del poder constituyente de la Federación, creemos, por las razones expuestas desde nuestra óptica antiformalosta del Derecho, que este requisito se cumple en España e Italia.

33 RUIPÉREZ ALAMILLO, J. División de Competencias.... p. 27.

${ }^{34}$ Cfr., De Vergottini, G. Diritto Costituzionale Comparato. Vol. 1 Padova. Milán 2007. pp. 391-392. 
España, Italia, Bélgica, México o Argentina y aunque en todos ellos existe un Senado la explicación no puede ser la que inspirada en concepciones contractualísticas acabamos de exponer.

La existencia de un Senado, así como el de procedimientos e instituciones que faciliten la cooperación intergubernamental (ultimo de los elementos de la lista más exhaustiva) son elementos convenientes y que facilitan el correcto funcionamiento de un Estado federal pero en modo alguno esenciales al mismo. Estos elementos serán, en nuestra humilde opinión, más necesarios en aquellos Estados federales en los que hay un mayor número de competencias concurrentes, y menos necesarios, sin embargo, allí donde la división constitucional de competencias responde a un criterio más claro.

Aunque el Profesor J. Ruipérez únicamente aborda en profundidad la discusión acerca de la Cámara de Representación territorial, confiando — lo afirma explícitamente- que el lector puede aplicar la misma lógica a los demás elementos discutibles, vamos a referirnos nosotros, de un modo casi telegráfico a los siguientes elementos, con la intención, simplemente, de ampliar la información.

La necesidad, o no, de la existencia de un árbitro es una cuestión un poco más compleja: lo que debe existir, y en esto coinciden todos aquellos que se han ocupado del asunto, y nosotros mismos lo hemos afirmado supra, es una garantía jurídica de que el legislador ordinario no puede dejar sin efecto la autonomía de los entes federados. Esta protección jurídica de su autonomía puede llevarse a cabo, que duda cabe, mediante el establecimiento de un Tribunal con facultades constitucionales, pero no necesariamente — pudiera hacerse, por ejemplo, mediante referéndum - Donde se hace de este modo, si se trata de un Estado, como España, que ha llegado al federalismo desde una situación anterior de centralismo político y territorial, este Tribunal puede convertirse, como ya hemos criticado en el epígrafe anterior, en protagonista indiscutible del proceso de descentralización. Sin embargo nosotros creemos que es más conveniente considerarlo, en todo caso, un corolario concreto de la garantía jurídica a la que antes nos referíamos, pues cualquier fórmula, establecida por el Poder Constituyente de un Estado cualquiera, que prestase de un modo razonable esta garantía, debería ser suficiente, desde el modo de razonar expuesto por nuestro autor, para considerarlo un Estado Federal.

Con mucho más motivo cabe afirmar esto del último elemento de la clasificación de D. Elazar. La propia exposición de este elemento (procedimientos e instituciones para «facilitar» la cooperación intergubernamental...) nos da la pista de que su ausencia, aunque criticable por muchos motivos, no puede impedir la consideración, por parte de la doctrina, de un Estado como Federal. De 
hecho, estos elementos acaban surgiendo, por la propia fuerza de los hechos, en los Estados Políticamente Descentralizados, ya sea estableciéndose a nivel formal (como, por ejemplo, la Conferencia Estado-Regiones en Italia ${ }^{35}$ o los convenios de colaboración entre las Comunidades Autónomas ${ }^{36}$ ), o a nivel informal (las Conferencias de Presidentes ${ }^{37}$ en España y Alemania). Su ausencia, sin embargo, no afecta a la forma política del Estado.

Habiendo sentado todas estas premisas podemos, recogiendo los elementos indicados, dar una simple definición de Estado Federal como aquel Estado que en su Constitución consagra el principio de autonomía y una distribución de competencias entre la Federación y los entes federados que viene asegurada por el principio de rigidez y supremacía constitucional. Definición a la que se ajusta, y esto es lo que a nosotros nos interesa ahora, España como Estado Federal de tipo disgregativo. Que ello resulte extraño, todavía, a teóricos y prácticos de la Política y el Derecho, debemos reconocer que nos cuesta comprenderlo, pues como trataremos de mostrar a continuación España cumpliría todos y cada uno de uno de los requisitos del tipo general Estado Federal. No sólo los de la clasificación más simplificada y que nos parece más correcta sino, también, incluso los de las clasificaciones más exhaustivas.

De este modo, es evidente que la Constitución española de 1978 dos niveles de gobierno, pues reconoce la autonomía política de las nacionalidades y regiones y se recoge el derecho de las Comunidades Autónomas a dotarse de su propia Asamblea Legislativa. Con menor fortuna, y esta es la verdadera fuente de conflictos, se recoge en los artículos 148 y 149 constitucionales una división de competencias entre las Comunidades Autónomas y el poder central. Por último, tampoco nadie de la doctrina constitucional española duda que España disponga de una Constitución rígida.

Aunque para nosotros sería suficiente con esto, no queremos dejar de constatar que, incluso desde clasificaciones como las de D. Elazar y K. Wheare se puede defender que España es un Estado Federal, aun cuando quepa denunciar serios problemas logísticos de funcionamiento. Está claro, así pues, y en primer lugar, que el Tribunal Constitucional ha sido un perfecto árbitro (y más que

35 Establecida por Decreto del 12 de octubre de 1983.

36 Para un estudio profundo de los mismos Cfr., RIAZ RICCI, R. Relaciones Gubernamentales de Cooperación. Ediar. 2009.

37 Cfr., TAJADURA TEJADA, J. «La conferencia de Presidentes» en AAVV. La cooperación intergubernamental en los Estados Compuestos. Institut d’Estudis Autonomics. Barcelona 2006. pp. 115-168 y también AJA, E. «La Conferencia de Presidentes en el Estado Autonómico». En AAVV Constitución, Estado de las Autonomias y Justicia Constitucional (Libro Homenaje al Profesor Gurmesindo Trujillo). Tirant Lo Blanch. Valencia 2005. pp. 823-840. 
eso incluso) en los conflictos entre las Comunidades Autónomas y el Gobierno Central, función que le es propia y atribuida por el art. 161 CE. Al margen de que sinceramente creemos, como ya hemos dicho, que sería imposible una reforma constitucional al margen de la voluntad de las Comunidades Autónomas, si nos dejáramos arrastrar a los argumentos más formalistas podríamos defender, en justicia, que las Comunidades Autónomas participan en la reforma constitucional a través del Senado, Cámara de Representación Territorial tal y como dispone el art. 69.1 CE (aun cuando en realidad su funcionamiento como tal es discutible) ${ }^{38}$. Por último, y en cuanto a los instrumentos formales e informales de cooperación (que existen y son abundantes) nos remitimos a lo ya indicado.

No queremos finalizar este epígrafe sin compartir la siguiente reflexión: Creemos poder afirmar que la Historia del concepto de Estado Federal se repite a lo largo del siglo XX. A principios de siglo advertía G. Jellinek que el concepto de Estado federal tuvo que adaptarse para que la doctrina admitiese a Alemania y Suiza, como nuevos tipos específicos de Estado Federal, junto a los Estados Unidos de América ${ }^{39}$. Ahora ocurre lo mismo con Estados como España o Italia. La primera parte, más general, del libro del Profesor J. Ruipérez que estamos comentando, es una clara toma de posición, coherente con su bibliografía anterior, pero expuesta de un modo más concreto, al respecto.

En relación con esto, y dada la pluralidad de matices que en formas descentralizadas de Estados podemos encontrar actualmente, parece lo más sensato, como por cierto también hacía el Profesor de la Universidad de Oxford, considerar que junto con los elementos esenciales al tipo federal existen otros posibles o recomendables ${ }^{40}$, pero no necesarios. En las páginas que siguen, de nuevo con el Profesor J. Ruipérez, repasamos los primeros.

${ }^{38}$ Cfr., por todos, CALZADA CONDE, R. y RUIPÉREZ ALAMILLO, J. «El Senado español: ¿auténtica cámara de representación territorial?». En Revista Jurídica de Navarra, n. ${ }^{\circ} 6$ (1988). pp. 123-131 y ROURA GOMEZ, S. «La Reforma Constitucional del Senado en la VIII Legislatura» En ROURA GOMEZ, S. y TAJADURA TEJADA, J. La Reforma Constitucional. Biblioteca Nueva. Madrid 2005. pp. 337-364. A mayor abundamiento, existe también un número monográfico (el 17) de TRC

39 JELLINEK, G. Teoría General del Estado. Traducción de F. de los Ríos. Comares. Granada 2002. p. 36.

${ }^{40}$ Cfr., WHEARE, K. op. cit. p. 199. 


\section{SOBERANÍA Y AUTONOMÍA, O SOBRE LA DISTRIBUCIÓN TERRITORIAL Y FUNCIONAL DEL PODER PÚBLICO COMO CARACTERÍSTICA BÁSICA DEL TIPO ESTADO FEDERAL}

Según el Profesor de la Universidade da Coruña si, como acabamos de ver, existen diferentes posiciones en la doctrina acerca de cuáles son los elementos caracterizadores del Estado Federal existe, sin embargo, unanimidad en la doctrina en considerar que en todo Estado Políticamente Descentralizado ha de verificarse un reparto territorial y funcional del poder político entre las dos instancias de decisión política que integran la Federación (lo hemos visto en el apartado anterior al mencionar, en todas las clasificaciones, la necesidad de que existieran «dos niveles de gobierno»). Un reparto — prosigue nuestro autorque en modo alguno puede interpretarse en el sentido de que tanto la organización política central como las organizaciones políticas regionales sean titulares de derechos de soberanía. Por el contrario, tal y como puso de manifiesto Friedrich y recogió el fundamento jurídico de la STC 4/1981 la soberanía únicamente pertenece al Pueblo Federal en su conjunto, mientras que a las colectividades miembros de un Estado Federal les corresponde un poder limitado de autonomía política (garantizada, como vimos, en último extremo, por la rigidez constitucional), que le garantiza una esfera competencial que no puede ser invadida por ninguna disposición del poder central ${ }^{41}$.

La tesis defendida requiere una profundización en la diferencia entre soberanía y autonomía porque, además, si bien es cierto, efectivamente, que la existencia de un reparto funcional y territorial del poder político es lo que esencialmente caracteriza un Estado federal y es, por tanto, el punto de partida de todas las construcciones doctrinales, no es posible afirmar tajantemente, sin embargo, que toda la doctrina comparta, hoy en día, la idea de que la soberanía, como poder absoluto, ilimitado e indivisible, pertenece en exclusiva al Pueblo federal mientras que la autonomía es el poder, limitado, que corresponde a las colectividades miembro. En este sentido no podemos olvidar que la tesis de la soberanía divisible, o co-soberanía, ha sido desde los orígenes mismos del surgimiento de la forma «Estado Federal», y sigue siendo en la actualidad, aun cuando el Profesor L. Pegoraro haya afirmado, y con razón, que empieza a ser recesiva, lugar común en la doctrina para explicar el Estado federal ${ }^{42}$. Conviene recordar que era el

${ }^{41}$ Cfr., para todo el párrafo, RUIPÉREZ ALAMILlO, J. División de Competencias... p. 33.

42 Cfr., PEGORARO, L. «Autonomía y descentralización en el derecho comparado: cuestiones metodológicas», En L. ESTUPIÑÁN ACHURY, G. GAITÁN BOHÓRQUEZ (comps.), El principio constitucional de autonomía territorial. Realidad y experiencias comparadas, Editorial Un. del Rosario, Bogotà D. C., 2010, pp. 3-23. 
propio J. Hamilton quien en El Federalista XXII explicaba la naturaleza de la Federación como una forma de Estado en que la soberanía se haya compartida entre las dos instancias de poder ${ }^{43}$. Su argumento se introdujo en Europa por A. De Toqueville, en su famosa La Democracia en América, y por eso ha gozado y goza aún de cierto predicamento aunque lo cierto sea que cada vez menos académicos están convencidos, como lo estaba G. Jellinek, de que los Länder, o cualquier ente federado parte de una Estado Federal, sean verdaderos y propios Estados. Para el Profesor de la Universidad de Heidelberg, las colectividades miembros tenían todos los elementos esenciales a un Estado, esto es, población, territorio y órganos estatales, aunque carecieran de un poder independiente que descanse en su propia voluntad ${ }^{44}$. Lo cierto es que él no sostiene que gocen de soberanía y, de hecho, en su definición de Estado Federal indica que el mismo consiste en una unión de Derecho Público de Estados no soberanos ${ }^{45}$, pero otros autores posteriores a él si han considerado que los Länder eran titulares de un poder soberano ${ }^{46}$, si bien limitado. Por ello es necesario que aclaremos porqué evidentemente no se pueden considerar tales. Desde el punto de vista del positivismo jurídico, es hasta cierto punto coherente reducir el problema de la soberanía a un problema de competencias, o de competencia sobre la competencia y, en última instancia, tal y como hizo $\mathrm{H}$. Kelsen, simplemente intentar erradicar este concepto afirmando, de modo relativista, que se trata de una mera hipótesis de trabajo ${ }^{47}$. Pero cuando descendemos de las nubes dogmáticas al terreno de la realidad política y social, coincidimos con G. Lucatello en afirmar que porque el jurista no debe dar la espalda a la realidad social, desde esta óptica, y también de acuerdo a una comparación de todos los Estados federales de su época, no se puede sino colegir que el Estado Federal es un verdadero Estado soberano ${ }^{48}$. Lo es porque el Estado en su conjunto es a quien corresponde modificar su Norma Fundamental así como destruirla y crear una nueva, sin depender más que de sí mismo, siendo sus normas generalmente válidas en todo el territorio, pues aun-

${ }^{43}$ HAMilton, A, MADiSON, J. y JAY, J. El Federalista. FCE. México. 1994. p. 127.

${ }^{44}$ Cfr., JELLINEK, G. op. cit. p. 649.

${ }^{45}$ Ibídem. p. 759.

46 Para una relación de los autores alemanes que sostienen la soberanía de los Länder, Vid., ARROYO GIL, A. «Cualidad estatal, soberanía, autonomía constitucional y competencia en la república federal de alemania: las constituciones de los "Länder" » En Revista d'Estudis Autonòmics $i$ Federals. N. ${ }^{\circ} 16.2012$. pp. 33 y ss, y su contestación a los mismos.

${ }^{47}$ Cfr., KELSEN, H. Il problema della sovranità e la teoría del diritto internazionale. Contributo per una dottrina pura del diritto. Traduzione Italiana di Agostino Carrino. Giuffrè. Milano. 1989. p. 13 y KELSEN, H. Teoría General del Derecho y del Estado. Traducción de Eduardo García Maynez. UNAM. México. 1995. p. 457.

${ }^{48}$ Cfr., LUCATELLO, G. Lo Stato Federale. CEDAM. Padova. 1967. p. 58. 
que es verdad que algunos Estados federales han establecido constitucionalmente la participación de los entes federados en el proceso de reforma constitucional, éste no es un requisito esencial para ser Estado Federal, si establecemos una comparación entre los Estados convencionalmente considerados como tales. Actualmente, la subsistencia de esta teoría de la co-soberanía se puede apreciar en que es lo que defienden no pocos académicos respecto del reparto de poder entre la Unión Europea y los Estados Miembros y lo que se deduce de la jurisprudencia del Tribunal de Justicia ${ }^{49}$.

Debemos distinguir pues, y como hemos anunciado, entre los conceptos de autonomía y soberanía que, sobre todo en el marco de los Estados federales creemos se usan a menudo con poco rigor. Simplificando, podemos decir con el Profesor I. Burgoa, que autonomía política es «darse sus propias leyes ${ }^{50}$, mientras que nosotros hemos definido la soberanía en otro lugar como «poder absoluto, indivisible, e ilimitado tanto en las formas como en el contenido de su voluntad, que es ejercido por un sujeto legítimo, con el fin de organizar los aspectos fundamentales del gobierno de una Comunidad Política»; definición de la que se deduce, sin demasiado esfuerzo, el derecho a llevar a cabo una revolución como la característica principal de este tipo de poder. Sin este poder, para no extendernos más en esta cuestión, es evidente, por lo tanto, desde nuestro punto de vista, que es de autonomía política, y no de soberanía, de lo que disfrutan las colectividades miembros de un Estado federal.

Partiendo entonces, de que sea la posibilidad de dirección política de los asuntos de su competencia sin injerencia por parte del poder central ni de ninguna otra esfera, y no la atribución de respectivos derechos de soberanía lo que, en definitiva, caracteriza un Estado federal y lo diferencia de un Estado unitario, prosigue el Profesor Ruipérez con dos precisiones de importancia al respecto.

La primera de ellas consiste en desmentir que un criterio para el entendimiento del Estado federal sea el mayor o menor número de competencias del que dispongan las colectividades miembros. Lo cierto - a juicio de quien suscribe estas líneas - es que en un Estado que de acuerdo con nuestro criterio pueda considerarse federal y en el que sin embargo el número de competencias en manos de las colectividades miembro sea bastante reducido puede crear insatisfacción, como ocurre por ejemplo en el caso de Argentina, al menos entre determinados sectores de la política y de la sociedad, pero ello no afecta a la natura-

49 Véase, en especial, la sentencia de 5 de febrero de 1963, Van Gend \& Loos, 26/62, Rec. p. 1.

50 Cfr. BURGOA, I. Diccionario de Derecho Constitucional, Garantías y Amparo. Editorial Porrua. México. 2000. pp. 59-61. 
leza de la forma de Estado. Obviamente, no se trata sólo de que sería un criterio científico, incluso bastante ridículo, el cuantificar las competencias de los entes federados y del poder central — lo que además implicaría una dificultad a mayores cuando hubiese que armonizar, para establecer una comparación, esas cantidades con las de los entes federados de otros Estados-, sino que además, como dice el Profesor de la Universidade da Coruña, la actual configuración de la República Federal alemana, de la que nadie duda que sea propiamente un Estado federal no se caracteriza precisamente porque los Länder gocen de una gran cantidad de competencias exclusivas. Evidentemente se refiere a la reforma del 28 de agosto de $2006^{51}$ que, en resumidas cuentas, vino a aumentar el poder federal en detrimento del rol del Bundesrat y los Länder.

Nosotros no creemos, humildemente, que se pueda hablar de una única razón que explique las tendencias centrípetas y centrífugas normales en la dinámica de un Estado federal cualquiera. De hecho, en su célebre trabajo de Derecho Constitucional Comparado, el Profesor M. García Pelayo indicaba ya que en las federaciones se da una tendencia al aumento de competencias estatales, y mencionaba varias causas posibles ${ }^{52}$. Entre ellas se encuentra una que es verdaderamente interesante y sobre la que hace especial hincapié nuestro autor y es el anhelo, en los modernos Estados Constitucionales, Democráticos y Sociales, de que los servicios públicos de prestación social que la federación garantiza por igual en todo el territorio sean cada vez más eficaces y eficientes. Esta posible causa merece especial consideración porque pone de manifiesto una -al menos potencialtensión entre el Estado social y el Estado federal cuando se da en él un alto nivel de descentralización. En momentos, sobre todo, como el actual, de crisis económica cabe efectivamente preguntarse acerca de si los entes regionales podrán, con recursos propios, sustentar por si mismos todos los servicios sociales que demanda la población, cuanto más, en momentos de depresión económica [que era, por cierto, otra de las causas, junto con la homogeneización política y social después de una vida en común, la complejidad de la estructura económica (de concentración de capital) y la pérdida relativa de importancia de los Estados miembros en la representación estatal que mencionaba el que fuera el primer Presidente del Tribunal Constitucional español en la obra citada].

Además, y para finalizar con esto, en los modernos Estados actuales, y por el desarrollo que ha experimentado el Derecho Internacional desde que García

${ }^{51}$ Ley Constitucional del 28 de agosto de 2006. BGB1. I, p. 2034, que entró en vigor el 1 de septiembre. Ley de acompañamiento de 5 de septiembre de 2006. BGB1. I, p. 2098.

52 Cfr., GARCÍA PELAYO, M. «Derecho Constitucional Comparado» en Obras Completas. CEPC. 2009. pp. 426 y 427. 
Pelayo escribía su conocida obra, no puede, tampoco, haber duda de que la condición de sujeto internacional, que corresponde únicamente a la federación, y las obligaciones en la sociedad de Estados que ello comporta, es un factor decisivo de centralización de los Estados federales. En concreto, tal y como apunta el Profesor Ruipérez en el trabajo que comentamos, el proceso de integración europeo debe ser un factor clave de centralización en los Estados europeos ${ }^{53}$. Sin embargo, aunque esto que, desde el año 2000, venía indicando el Profesor de la Universidad de la Coruña que pasaría es lo que, en buena lógica jurídica hubiera ocurrido si la Unión Europea se estuviese construyendo desde los presupuestos del constitucionalismo democrático y social — pues nadie puede, creemos, dudar, que si son únicamente los Estados los que son sujetos de Derecho Comunitario, y son por tanto ellos los únicos responsables ante la Unión Europea de los eventuales incumplimientos del Derecho de la Unión, al margen de a quien corresponda, en el plano interno la competencia, esto supone un evidente factor de centralización- lo cierto es que por ser la Unión Europea «hija» de la globalización ${ }^{54}$ y la postmodernidad, casi podríamos decir que sucede más bien lo contrario. De esta forma, desde premisas intelectuales que niegan que pueda defenderse una única racionalidad en el proceso de integración supranacional, y la renuncia a crear conceptos únicos o a identificar un único demos europeo como sujeto de derechos, de la misma forma que, la regionalización es la otra cara de la globalización (Höffe ${ }^{55}$ o, entre otros, Castells ${ }^{56}$ ) por paradójico que pueda resultar, el proceso de integración europea y la regionalización de algunos Estados (España, Italia, Gran Bretaña e, incluso, Francia), aunque de signo contrapuesto, se retroalimentan, recibiendo influencias recíprocas ${ }^{57}$. No es este el lugar para entrar en una exposición pormenorizada de este hecho, por eso nos conformaremos tan sólo con indicar, que cabe apreciar estas influencias recíprocas a las que nos referimos tanto a nivel intelectual y doctrinal (p. ej. en la identificación

53 Cfr., RUIPÉREZ ALAMILLO, J. op. cit. pp. 121 y 122. Este asunto fue desarrollado con más detalle en La Constitución Europea y la Teoría del Poder Constituyente. Algunas reflexiones desde el Derecho Político.

${ }^{54}$ En general, sobre el fenómeno de la globalización y sus consecuencias para el Derecho Constitucional Cfr., DE VEGA, P. Mundialización y Derecho Constitucional. La crisis del principio democrático en el constitucionalismo actual». Revista de Estudios Políticos, n. ${ }^{\circ} 100$ (1998).

55 Vid., HÖFFE, O. «Estados nacionales y Derechos Humanos en la era de la globalización» (versión castellana de R. R. Araujo y J. C. Velasco. En Isegoria, n. ${ }^{\circ} 22$ (2000).

56 Vid., CASTELLS, M. «Globalización, Estado, y Sociedad Civil: el nuevo contexto histórico de los derechos humanos» En Isegoría, n. ${ }^{\circ} 22$ (2000).

57 Para una primera aproximación de este fenómeno me remito a FONDEVILA MARÓN, M. «El Proceso de Integración Europea en relación con la Descentralización en los Estados Miembros». En Anuario da Facultade de Dereito da Universidade da Coruña. N. ${ }^{\circ} 14$. pp. 385 a 421. 
entre soberanía y competencia de la competencia) como a nivel normativo (por ejemplo, en España las declaraciones estatutarias de derechos están claramente influenciadas por la Carta de Niza).

De todas formas, en nuestra humilde opinión, aquella tendencia que mencionaba el Profesor García Pelayo habría hoy que matizarla con la experiencia de los nuevos Estados federales, los que surgen como un proceso de disgregación de Estados otrora unitarios. En ellos, creemos poder afirmar nosotros se ha dado hasta el momento más bien la tendencia contraria: la de una paulatina descentralización del poder político desde la federación a los entes regionales. Por eso, con acierto - a nuestro juicio_ pone de manifiesto el Profesor J. Ruipérez en la obra que comentamos, que se debe comprender, con A. La Pérgola, que estas tendencias centrípetas y centrífugas que se dan, en sentido opuesto, en un Estado Federal, convergen de algún modo ${ }^{58}$, pues estando el Estado Federal, como cualquier otro, sometido al devenir histórico, la reforma constitucional y, cuando sea posible, la mutación constitucional habrán de satisfacer el juego de tendencias, sin que el reparto competencia entre la federación y los entes federados pueda quedar, de una sola vez, petrificado en el texto de la Constitución.

Por ello, aunque la forma del reparto territorial y funcional del poder público no afecte a la naturaleza del Estado federal sí existe una competencia de especial trascendencia que necesariamente tendrá que residir los órganos de la Federación. Nos referimos a la llamada «competencia sobre la competencia», cuyo tratamiento en la obra objeto de estudio, exponemos a continuación.

\section{DE LA KOMPETENZ-KOMPETENZ Y SUS LÍMITES}

La «competencia sobre la competencia», término que fue acuñado por la doctrina decimonónica iuspublicista alemana es definida por el Profesor de la Universidad de La Coruña como «la facultad que se atribuye a alguno de los entes políticos territoriales que integran el Estado Federal para decidir en última instancia sobre su propio ámbito de poder condicionando, y esto es lo importante, con su decisión la extensión de la competencia de los otros entes políticos en los que se divida aquel Estado» ${ }^{59}$. De esta definición es menester llamar la atención sobre el hecho de que lo que puede hacer el titular de la kompetenz-kompetenz es condicionar la extensión de la autonomía de la que disfrutan los demás entes político-territoriales, porque lo que sostendrá nuestro autor en los dos últimos capítulos de su obra es, precisamente, que la autonomía política en sí es un

${ }^{58}$ Cfr., RUIPÉReZ ALAMILlO, J. División de Competencias... p. 49.

59 RUIPÉREZ ALAMILLO, J. División de Competencias... p. 59. 
límite, si acaso implícito, al ejercicio de esta competencia. Que ello sea así se explica porque no cabe confundir, como hacen algunos autores, la «competencia sobre la competencia» con soberanía. Si no, no tiene sentido utilizar el concepto de «competencia» que, como expusiera en su momento C. Schmitt, apela necesariamente a un concepto jurídico limitado.

Es interesante, a nuestro juicio, detenernos un poco en esta cuestión porque aquellos autores que, como por ejemplo entre nosotros, Miguel Herrero, identifican expresamente como soberano al que ejerce la «competencia sobre la competencia», necesariamente, como él, tendrán que defender en el seno de un Estado Federal que la soberanía, es decir, esa competencia sobre la competencia, es divisible, y se encuentra en poder tanto de la organización política central como de los Estados miembros ${ }^{60}$. No insistiremos más sobre lo insostenible de la doctrina de la soberanía divisible, que como hemos dicho es hoy minoritaria, pero lo que nos interesa es poner de manifiesto, sin embargo, que porque la «competencia sobre la competencia» (cuando se asimila, como hace el positivismo jurídico desde Jellinek), la soberanía divisible, así como la pura y simple negación del concepto de soberanía, tienen postulados similares y conducen, por lo tanto, a las mismas conclusiones. La razón de esta afirmación se basa en los siguientes argumentos: Primero, se comprende fácilmente que los que defienden la tesis de la soberanía divisible, porque confunden soberanía y competencias, necesariamente tienen que considerar verdaderamente soberano a aquel que ejerce la competencia de la competencia. Segundo, resulta también evidente, que en el marco de una Comunidad de carácter federal, aquél que, al estilo de un H. Kelsen, niegue la idea de soberanía como concepto útil para comprender las relaciones reales de poder existentes tanto en el seno de los Estados como en el ámbito de la Comunidad Internacional, acabará afirmando (explícita o implícitamente), al igual que los hablan de soberanía divisible y de soberanía como competencia de la competencia, que en las dos esferas de poder perfectamente reconocibles cada uno podrá establecer normas jurídicas válidas en desarrollo de la norma de atribución competencial. Tercero y último, paralelamente, como es lógico, aquellos que afirmen la soberanía divisible negarán, en buena lógica, a ambas partes que se predican «soberanas» el derecho a la revolución en el sentido de modificar unilateralmente las normas fundamentales por las que se rige la Comunidad, si con ello alteran las competencias del otro ordenamiento jurídico. Negarán, en el caso de la Federación, la posibilidad del Pueblo en su conjunto

${ }^{60}$ Vid., HERRERO Y RODRIGUEZ DE MIÑON, M. «A vueltas con la soberanía (la soberanía en la Constitución» en Anales de la Real Academia de Ciencias Morales y Políticas. N. ${ }^{\circ} 84$ (2007). p. 326. 
de dotarse de una nueva Constitución al margen de la voluntad, aunque sólo sea, de los órganos representativos de un Estado miembro, y negarán, por supuesto, a las colectividades miembros el derecho de secesión por no tener ellas tampoco el derecho de modificar unilateralmente la norma constitucional. Al privar al poder soberano de su atributo más natural, podemos decir también, niegan el concepto mismo de soberanía. Las tres doctrinas llevan a resultados absurdos y contradictorios incluso dentro de la lógica de su propio paradigma epistemológico, y aunque no vamos a seguir desarrollando esta cuestión nos preguntaremos, únicamente a título de ejemplo, cómo podrá un positivista que defienda la sustitución del concepto de soberanía por el de «competencia sobre la competencia» (aunque el fondo esté defendiendo cosas similares a los que hablan de co-soberanía) defender la ilegitimidad de una reforma constitucional, llevada a cabo por los órganos de la federación, siguiendo todas los requisitos formales previstos en la Constitución, que tenga como objeto desposeer al otro sujeto de la «competencia sobre la competencia» de la misma.

Por ello nos parece más lógica, consecuente y sostenible la tesis del Profesor Ruipérez en la obra objeto de estudio. Trataremos de resumirla a continuación indicando el titular, el objeto, y los límites de su ejercicio.

En cuanto al primer punto, indica el Profesor de la Universidad de La Coruña que «es la organización política central la que, por su propia naturaleza, esto es, en cuanto sujeto político que actúa en todo el territorio estatal y que, aunque es distinto y, en cierto modo, contrapuesto a las organizaciones políticas regionales, no es, empero, enemigo de ellas, se presenta como la única instancia de decisión política que sería capaz de llevar a cabo la defensa de los intereses comunes y generales de la Comunidad Política. Y es, en efecto, a ella, a quien le corresponde tal tarea, toda vez que, dicho sea con el mayor de los respetos, y desde la más elevada objetividad, lo que a las organizaciones políticas regionales interesa no es tanto, o, al menos, no directamente, el interés general cuanto la satisfacción de sus propios intereses o fines» ${ }^{61}$.

Lo que este sujeto de Derecho puede hacer, en el legítimo ejercicio de la «competencia sobre la competencia» en la teoría del Profesor Ruipérez, basada en la obra de Friedrich, no es otra cosa que llevar a cabo el procedimiento de reforma constitucional, cuando sea jurídicamente necesario y, por tanto, políticamente conveniente (P. De Vega ${ }^{62}$. Esto sí merece, al menos, de dos comentarios por nuestra parte: En algunos Estado Federales, como es el caso paradigmático de los Estados Unidos (art. V) se ha decidido soberanamente, en el texto de

\footnotetext{
${ }_{61}^{6}$ RUIPÉREZ ALAMILLO, J. División de Competencias... pp. 104-105.

${ }^{62}$ Ibídem. p. 112.
} 
la Constitución, que los proyectos de reforma constitucional deban ser aprobados por los Estados. En otros casos, como en Alemania, basta la aprobación de las ambas cámaras del Parlamento (art. 79) si bien, en este caso, la división en Länder de la República Federal constituye una cláusula de intangibilidad. En cualquier caso, lo importante en este punto es que, aunque la decisión del Poder Constituyente de hacer co-participes de las reformas constitucionales a las entidades federativas puede ser, una vez más, políticamente conveniente para la convivencia y, en definitiva, el funcionamiento del Estado federal, no es imprescindible, por las razones expuestas, que sea así. En la mayoría de Estados las colectividades miembro participan en los proyectos de reforma a través de su representación en el Senado, o cámara de representación territorial, que a pesar de su composición es, evidentemente, un órgano federal. Pero como hemos visto un Estado Federal ni siquiera tiene porqué contar con una Cámara de este tipo. Otro aspecto importante digno de resaltar, pues sin él no se entiende qué teoría del Estado Federal o políticamente descentralizado defiende nuestro autor, es que si corresponde el poder de reforma únicamente a la federación es porque este, tal y como ha defendido en un reciente trabajo sobre los límites a la reforma constitucional $^{63}$, esta es una operación ontológicamente limitada, aun cuando no conste así, como en el caso de Alemania, en el texto constitucional.

En concreto, por lo que a la forma territorial del Estado se refiere, el límite al que ve sujeto el poder de reforma en un Estado Federal o Políticamente Descentralizado no es otro, entonces, que aquellas modificaciones que despojarían totalmente a las colectividades miembros de toda competencia, convirtiendo entonces a la federación en un Estado Unitario o, en un extremo opuesto, donde seguir otorgando competencias a los entes federados supondría convertir el Estado Federal en una Confederación de Estados ${ }^{64}$. La forma de Estado se convierte así en un límite material implícito - con las dificultades interpretativas que ello pueda suponer - a todo poder constituido, y por lo tanto, limitado, de reforma.

Cuestión distinta es si esta teoría general del Estado Federal tiene una fácil aplicación en la práctica constitucional española. Su postura, en síntesis, es que existen dificultades derivadas, principalmente de dos hechos: Primero, y como es de sobra conocido, porque la Constitución de 1978 no cerró, como hubiera sido deseable, un reparto competencial claro y preciso entre las Comunidades Autónomas y la organización política central sino que, al contrario, apenas se consiguió en el momento constituyente un «compromiso apócrifo» (P. de Vega) sobre el modelo competencial y fue en virtud del llamado «principio dispositi-

${ }^{63}$ Concretamente en el n. ${ }^{\circ} 30$ de Teoría y Realidad Constitucional.

${ }^{64}$ Cfr., RUIPÉREZ ALAMILlO, J. División de Competencias... pp. 129 y ss. 
vo» como las Comunidades Autónomas fueron configurando, cada una a su ritmo, su marco competencial. Un ejemplo concreto de esta indeterminación competencial puede apreciarse en el artículo 149.3 de nuestra Constitución, donde a diferencia de otros Estados Federales, no se reserva, sin más, una competencia residual de los entes regionales para todo lo no atribuido al poder político central sino que las Comunidades Autónomas podrán legislar sobre lo no reservado al poder central únicamente si han consignado dicha competencia en sus respectivos Estatutos de Autonomía. Segundo, porque una interpretación abusiva y extensiva de este principio dispositivo por parte de los nacionalismos periféricos, consagrada, por cierto, por el Tribunal Constitucional, lleva necesariamente a que la indeterminación del modelo competencial a la que nos referimos sea una fuente permanente de conflictos territoriales.

Ningún comentario a mayores vamos a hacer nosotros en este trabajo respecto al primer punto mencionado, pues creemos que en ello coincide la unanimidad de la doctrina, y sería por tanto un ejercicio superfluo. Pero si nos interesa hacer algunas precisiones respecto de la resucitación, por la doctrina y la jurisprudencia, del principio dispositivo y de cómo ello dificulta la ordenación definitiva del modelo territorial.

Aunque hubiese sido razonable pensar, que al alcanzarse el mismo techo competencial para todos en $1992 \mathrm{el}$ principio dispositivo, consagrado en nuestro desafortunado Título VIII de la Constitución, había quedado enterrado ${ }^{65}$, las ansias del nacionalismo catalán por diferenciarse y — dicho sea con todos los respetos - la falta de determinación de nuestro Tribunal Constitucional para, en el cumplimiento de la función que tiene encomendada, oponerse a las pretensiones más elevadas, y que excedían el marco de la Constitución de 1978, supusieron la resucitación de este principio, consagrado de nuevo gracias a las SSTC 247/2007 y 31/2010. Como acertadamente, a nuestro juicio, denunció la doctrina, en la primera de las sentencias mencionadas, aun cuando no existía necesidad de ello, sorprendió a casi todos el supremo intérprete constitucional esgrimiendo la tesis de que, al parecer, la Constitución había dejado «desconstitucionalizada» la materia competencial, sin precisar con exactitud las materias sobre las que se proyecta las distintas competencias mencionadas y que, en consecuencia, los Estatutos de Autonomía podían realizar una interpretación de la Carta Fundamental en este sentido ${ }^{66}$. Con razón, algún sector de la doctri-

${ }^{65}$ Cfr. PÉREZ ROYO, J. «La reforma del Estado Autonómico» en Ferrer McGregor, E y Zaldivar Lelo de Lárrea, A. op. cit. Marcial Pons. México, 2008, Tomo XI, p. 226.

66 Vid., fundamento jco. 7..$^{\circ}$ de la STC 247/2007 y, para una crítica, Fernández Farreres, G. ¿Hacia una nueva doctrina constitucional del Estado Autonómico? (Comentario a la STC 247/2007 de 
na manifestó que en esta sentencia lo que hacía el Tribunal era, de cara la futura sentencia sobre el Estatuto de Cataluña, preparar una mutación constitucional que permitiese encajar (al menos la mayor parte de) un texto sobre el que todos los estudiosos de la Ciencia constitucional abrigaban serias dudas, dentro del edificio constitucional ${ }^{67}$. La segunda de las sentencias mencionadas más arriba matiza algunas expresiones problemáticas de la primera pero, en definitiva, en sus fundamentos jurídicos 59 y 64 reitera una doctrina constitucional desde nuestro punto de vista criticable, y tendente permitir, a priori, que los Estatutos de Autonomía asuman casi cualesquiera competencia en su articulado, incluyendo las recogidas en el artículo 149.1 CE.

Nosotros — de la mano del Profesor Ruipérez — hemos dicho que el marco competencial podía, efectivamente, estar fijado con mayor precisión en el texto constitucional, pero esta norma tampoco permitía, a nuestro juicio, lo que el Tribunal Constitucional ha permitido en una interpretación más que extensiva del art. 149.3 CE, esto es, que los Estatutos de Autonomía se atribuyan competencias que estaban, en exclusiva, reservadas al Estado, con el pretexto de que no se fijan con precisión todos los apartados y sub-materias que comprenden ${ }^{68}$.

\section{CONCLUSIONES}

Los españoles asistimos atónitos a un proceso de integración europea y a un conato de proceso independentista por parte de la Comunidad Autónoma de Cataluña que, paradójicamente, coinciden en el tiempo. Lo que hemos intentado demostrar en este estudio es que ni la doctrina ni los prácticos de la Política y el Derecho parecen preparados para afrontar tales desafíos teniendo en cuenta las dudas y vacilaciones existentes respecto de la forma territorial del Estado de las Autonomías.

Comprender las particularidades de nuestra forma territorial de Estado es el punto de partida para una reforma del mismo, dado que la práctica unanimidad de la doctrina asume lo desafortunado e inconcluso de nuestro Título VIII constitucional. La obra que hemos comentado fija las bases, indica quienes son los

12 de Diciembre, sobre el Estatuto de Autonomía de la Comunidad Valenciana). Cuadernos Civitas. Navarra, 2008. pp. 75-78.

${ }^{67}$ Cfr. CANOSA USERA, R. «Preparando una mutación constitucional. Comentario a la STC 247/2007, de 13 de diciembre. En Teoría y Realidad Constitucional. n. ${ }^{\circ} 22$ (2008).

68 Para mayor abundamiento: FONDEVILA MARÓN, M. «Los Nuevos Estatutos de Autonomía en el marco del Derecho Constitucional Comparado» en Boletín Mexicano de Derecho Comparado, n. ${ }^{\circ} 133$ (2012). pp. 13-54. 
responsables de llevarla a cabo y cuáles son los limites materiales, explícitos e implícitos que deben respetar. Evidentemente son los prácticos de la política los que deben arbitrar las soluciones.

En este humilde trabajo lo único que por nuestra parte hemos intentado es demostrar que División de Competencias y Forma Territorial del Estado responde a un debate actual y presente —razón por la cual hemos tratado de contrastar las principales ideas con la doctrina y la jurisprudencia - puesto que, hoy más que nunca, las fuerzas centrífugas y centrípetas que a menudo se dan en un Estado Políticamente Descentralizado tienen un papel relevante en la política diaria.

Title:

DIFICULTIES FOR DEVELOPING A THEORY ON THE STATE OF AUTONOMIES. (ON «DIVISIÓN DE COMPETENCIAS Y FORMA TERRITORIAL DEL ESTADO» DE JAVIER RUIPÉREZ ALAMILLO).

\section{Sumary:}

I. Introduction: Socio-political context with which this essay is in line. II. The state of autonomies and its indeterminate political form in the Constitution of 1978. III. On the characterization of the elements of the «Federal State» type. Sovereignty and autonomy or on the political and territorial distribution of public power as basic feature of federal state type. V. On the «kompetenz-kompetenz» and its limits. VI. Conclusions.

\section{Resumen:}

Este ensayo aborda, a propósito del reciente libro del Profesor J. Ruipérez División de Competencias y Forma Territorial de Estado las dificultades que, desde el origen del Estado de las Autonomías, se ha encontrado la clase política y académica para comprender correctamente la naturaleza de la forma de Estado. La tesis sostenida es que el Estado de las Autonomías es un tipo concreto de Estado Federal, aun cuando sería conveniente una reforma constitucional que clarificase el reparto competencial entre el gobierno central y las Comunidades Autónomas.

\section{Summary:}

This essay deal with, referring of recent book of Professor J. Ruipérez División de Competencias y Forma Territorial del Estado the difficulties that, since the origin of the State of Autonomies, political and acade- 
mic class have found to a correctly understanding of the nature of its political form. The thesis is that the State of Autonomies is a specific type od Federal State, even if it would be appropriate a constitutional reform in order to clarify distribution of powers between the central government and the autonomous communities.

\section{Palabras Clave:}

Estado de las Autonomías; Estado Federal; Competencias; Soberanía; Autonomía.

\section{Keywords:}

State of Autonomies; Federal State; Competences; Sovereignty; Autonomy. 